\title{
ESTUDIO TECNO-MORFOLÓGICO DE PALAS Y/O AZADAS LÍTICAS DEL SECTOR NORTE DEL VALLE DE HUALFÍN (CATAMARCA, ARGENTINA)
}

\section{TECHNO-MORPHOLOGICAL STUDY OF LITHIC SHOVELS AND/OR HOES ON NORTHERN HUALFIN VALLEY (CATAMARCA, ARGENTINA)}

\author{
Emiliano Bentivenga ${ }^{1}$, Julieta Lynch², Virginia Lynch ${ }^{3}$ \\ ${ }^{1}$ CONICET - División Arqueología, Museo de La Plata. Paseo del Bosque s/n La Plata (1900), \\ Buenos Aires, Argentina. Email: emilianobenti@hotmail.com \\ ${ }^{2}$ CONICET - División Arqueología, Museo de La Plata. Paseo del Bosque s/n La Plata (1900), \\ Buenos Aires, Argentina. Email: julietalynch@gmail.com \\ ${ }^{3}$ Universidad Nacional de La Plata, UNLP, División Arqueología, Museo de La Plata. Paseo del \\ Bosque s/n La Plata (1900), Buenos Aires, Argentina. Email: lynchvirginia@gmail.com
}

Presentado: $14 / 07 / 2020$

Aceptado: 04/11/2020

\section{Resumen}

La presencia de palas y/o azadas líticas fue registrada en distintas regiones del Noroeste Argentino y comúnmente relacionada con el trabajo agrícola en contextos de ocupaciones formativas y tardías. Sin embargo, estos artefactos no han sido reportados hasta el momento para el Valle de Hualfin (Catamarca, Argentina). El objetivo principal de este trabajo es analizar, desde una perspectiva tecno-morfológica, las palas y/o azadas procedentes del sitio Villavil 1, al norte del valle. Para el análisis se seleccionaron ciertas variables tecnomorfológicas y se compararon con los datos disponibles en la región. A partir del estudio desarrollado se identificaron las técnicas de manufactura de estos artefactos que, en comparación con el resto del conjunto lítico, evidencian una mayor inversión de trabajo en su producción, lo que señala la importancia de la actividad agrícola para las sociedades formativas y tardías que habitaron la región.

Palabras clave: Palas y/o azadas líticas, Noroeste Argentino, Agricultura, Períodos Formativo y Tardio

\section{Abstract}

The presence of shovels and/or hoes was recorded in several regions of Northwestern Argentina, and mainly associated with agricultural activities developed in formative and late contexts. Nevertheless, so far these artifacts have not been reported in the Hualfin Valley (Catamarca, Argentina). The main aim of this article is to analyse, from a technomorphological perspective, the shovels and/or hoes coming from Villavil 1 site, located in the north of the valley. To analyse these artefacts, we chose some techno-morphological 
variables and compared the results with the available data in the region. From the study carried out, we identified the manufacturing techniques of these artefacts which, compared with the rest of the lithic assemblage, show a greater production effort invested. This indicates the significance of the agricultural activities to formative and late societies that inhabited the region.

Key words: Lithic shovels and/or hoes, Northwestern Argentina, Agriculture, Formative and Late Periods

\section{Introducción}

La producción de alimentos por parte de poblaciones humanas ha sido el foco de interés de diversas investigaciones arqueológicas a nivel global (Bogaard 2005; Capparelli et al. 2011; Childe 1998 [1936]; Fairbairn 2005; Kolata 1985; por mencionar algunos ejemplos), al igual que las investigaciones desarrolladas en torno a los elementos de labranza utilizados por las poblaciones agropastoriles (Clemente y Gibaja 1998; Kvietok 1987; Palomo et al. 2013; Sonnenfeld 1962; Xie 2018; Yang et al. 2016). En este sentido, creemos que profundizar los estudios sobre las herramientas líticas utilizadas, permite recuperar valiosa información de las prácticas agrícolas prehispánicas en diferentes regiones del mundo.

Para el Noroeste Argentino (NOA), la producción agrícola ha sido propuesta como la principal actividad económica durante los períodos Formativo (ca. 550 a.C. - 900 d.C.) y de Desarrollos Regionales (ca. 900 - 1430 d.C.), mayormente en la región Valliserrana. La presencia de obras de infraestructura prehispánica como andenes y terrazas de cultivo, canales de riego, silos, entre otras; así como la identificación de restos botánicos, constituyen las principales evidencias arqueológicas a partir de las que se ha inferido el desarrollo de actividades productivas (González 1955, 1979; Raffino 2007; Sempé 1999, 2005). Si bien se ha reportado la presencia de hojas de palas y/o azadas líticas en distintos contextos arqueológicos, su estudio no fue sistemático (Alfaro de Lanzone 1969; Bennett 1948; Márquez Miranda 1939). Posiblemente, dicho sesgo esté relacionado con la mayor relevancia que se le dio tradicionalmente a la arquitectura y la cerámica, en detrimento de otras materialidades presentes en el registro arqueológico del NOA.

No obstante, en las últimas décadas han proliferado las investigaciones focalizadas en distintas tecnologías líticas de sociedades, tanto formativas como tardías del NOA (Álvarez 2004; Avalos 2003; Carbonelli y Gaál 2015; Chaparro 2008-2009; Elías 2012; Escola 2000; Flores 2012, 2014; Flores y Wynveldt 2009; Gaál 2011a, 2011b; Miguez et al. 2009; Sprovieri y Baldini 2007, entre otros), y los estudios de palas y/o azadas no fueron la excepción, llevándose a cabo análisis de ejemplares procedentes de la Puna catamarqueña (Gastaldi 2008; Haber y Gastaldi 2006; Pérez 2010, 2012), jujeña (Avalos 1998; Pérez 2010), como así también de la Quebrada de Humahuaca (Avalos 1998; Fabron 2012; Leoni et al. 2014) y, para el área Valliserrana, del norte de la provincia de La Rioja (Borgo 2014). 
Las palas y/o azadas están conformadas por una hoja lítica y un mango, posiblemente de madera, unidos entre sí por una atadura de cuero o fibra vegetal (Haber y Gastaldi 2006). Pérez (2010) considera que el enmangue es una práctica vinculada a la búsqueda funcional del instrumento y su preparación para tal fin implica una anticipación al uso, lo cual se asocia a una estrategia tecnológica conservada.

En el registro arqueológico normalmente solo se preservan las hojas, y Pérez (2012) consiguió identificarlas a partir de análisis tecno-morfológicos y traceológicos integrados en un programa experimental. De esta manera, la autora estableció la localización, distribución y orientación de rastros como estrías, pulidos, filos redondeados y microfracturas. Los resultados mostraron una diferenciación de las estrías localizadas en los bordes de las partes activas y las ubicadas en las zonas de enmangue.

A partir de dichos estudios (Pérez 2007, 2010, 2012) estos artefactos son integrados en la clasificación tipológica de Aschero (1975), dentro del grupo de palas y/o azadas líticas manufacturadas por lascado, y definidos como instrumentos formatizados por lascados directos de retalla y retoque bifacial marginal en todo su perímetro, con una morfología general predominantemente semielíptica. Haber y Gastaldi (2006) describen la conformación de filos a partir de retalla y retoque. La primera es unifacial y es utilizada para el adelgazamiento de la sección de enmangue y la confección de las aletas. El retoque es bifacial en toda la pieza con una dirección de lascado escamoso irregular y también regular, en menor proporción, conformando un bisel doble asimétrico. En ciertos casos se registraron aletas y pedúnculos con filos embotados (Haber y Gastaldi 2006; Pérez 2010, 2012). Algunos de los artefactos asignados a este grupo tipológico presentan filos naturales y biseles diferenciados por pulido (Avalos 1998; Fabron 2012; Gastaldi 2008; Pérez 2010, 2012).

En cuanto a las formas base empleadas, las piezas procedentes de las áreas puneñas y Quebrada de Humahuaca están confeccionadas sobre lajas de origen volcánico, basalto y andesita (Avalos 1998; Gastaldi 2008; Pérez 2010, 2012), así como sobre esquistos (Avalos 1998; Fabron 2012; Leoni et al. 2014). A su vez, se diferencian dos morfologías principales, por un lado, ejemplares apedunculados, y por otro, piezas con secciones diferenciadas en cuerpo, inflexión cuerpo-pedúnculo y pedúnculo. Si bien los conjuntos mencionados presentan cierta variabilidad interna, Pérez (2012) adscribe a las piezas procedentes de Casa Chávez Montículos (Puna catamarqueña) a un mismo estándar morfológico, que se manifiesta en la serie técnica, forma general, ángulos y espesores de las piezas.

Asimismo, hay reportes de piezas con características que se diferencian de las anteriores, pero que igualmente han sido identificadas como palas y/o azadas. Un ejemplo es el estudio tecno-morfológico que presenta Borgo (2014) sobre artefactos con borde perimetral formatizado procedentes de La Cuestecilla (norte de La Rioja). Se trata de ejemplares confeccionados sobre lajas metamórficas, filitas y pizarras, con bordes perimetrales formatizados donde se distinguen potenciales huellas de uso. 
Fuera de la región del NOA, Salgán y Pompei (2020) presentaron el estudio de palas, azadas y/o cuñas líticas asociadas a la fuente de obsidiana de cerro El Peceño, al sur de la provincia de Mendoza. Estos artefactos están confeccionados sobre lascas de arenisca y pelita con formatización marginal, tanto unifacial como bifacial, cuyas morfologías son circular irregular y triangular. Por el contexto de hallazgo, se interpretó que se habrían empleado para la extracción de materias primas del suelo.

A pesar de los antecedentes mencionados en distintos sectores del NOA, los estudios realizados sobre palas y/o azadas en el Valle de Hualfín continúan siendo escasos. Valencia y colaboradoras (2009) registraron el hallazgo de restos de un ejemplar de pala de madera (Prosopis) en Campo de Carrizal, al sur del valle. Mientras que, en las proximidades de esta área, en el Valle de Quimivil, Giovannetti (2009) halló restos enteros y fracturados de palas y/o azadas líticas del sitio Los Colorados, en distintos sectores que habrían funcionado como áreas de cultivo.

A partir de lo mencionado anteriormente, en este trabajo se presenta el estudio, desde una perspectiva tecno-morfológica, de palas y/o azadas líticas procedentes del sitio Villavil 1, emplazado al norte del valle (Catamarca, Argentina). Para ello, se propone describir morfológicamente dichos instrumentos, realizar un estudio comparativo con aquéllos identificados en otras áreas del NOA y analizar el grado de inversión de trabajo requerido para su producción. De esta manera, se espera profundizar el conocimiento que se tiene hasta el momento de las prácticas agrícolas desarrolladas por las sociedades formativas y tardías del área de estudio.

\section{Consideraciones teóricas}

Para abordar los objetivos enunciados se considera el concepto de organización tecnológica como "el estudio de la selección e integración de estrategias para confeccionar, usar, transportar y descartar instrumentos y materiales necesarios para su mantenimiento" (Nelson 1991: 57). Dichas estrategias se encuentran ligadas a las condiciones tanto ambientales como sociales, ya que son producto de la interacción de los grupos humanos con su entorno, en relación con sus necesidades y prioridades particulares.

Los distintos tipos de estrategias llevadas a cabo por las sociedades involucran las diferentes etapas de confección, uso, transporte y descarte. Las estrategias conservativas responden a una mayor anticipación y preparación ante situaciones adversas, mientras que las expeditivas minimizan el esfuerzo tecnológico ante condiciones predecibles (Nelson 1991). A su vez, las diferentes maneras de implementar la conservación y la expeditividad están entrelazadas, y determinan materialmente la forma de los artefactos, así como la composición de los conjuntos, contribuyendo a la complejidad del registro material (Escola 2004; Nelson 1991). 
Diferentes autores han relacionado el tipo de estrategia tecnológica con la inversión de trabajo requerido en la producción lítica (Andrefsky 1994; Hocsman y Escola 2006-2007; Parry y Kelly 1987). De este modo, las estrategias conservativas requieren una mayor inversión de trabajo, mientras que las expeditivas insumen un menor esfuerzo de producción. Por lo tanto, identificar el nivel de trabajo empleado en la confección de un conjunto instrumental, permite caracterizar la estrategia tecnológica involucrada.

Hasta el momento, a partir de los estudios de tecnología lítica llevados a cabo en los sitios Villavil 1 y 2, al norte del Valle de Hualfín, se ha propuesto el empleo de estrategias principalmente expeditivas para esta área del valle. Dichas estrategias se corroboran por la baja producción de artefactos formatizados, la predominancia de diseños que requieren una baja inversión de trabajo en su confección y la utilización de materias primas mayoritariamente locales (Bentivenga y Ríos Malan 2020; Lynch y Lynch 2016; Lynch et al. 2016). El siguiente trabajo amplía el conocimiento sobre estas estrategias tecnológicas desarrolladas en el primer sitio, a partir del estudio del conjunto de palas y/o azadas recuperadas en diferentes sectores.

\section{Zona de investigación: sitio Villavil 1}

El Valle de Hualfín es parte de la región geológica Sierras Pampeanas Occidentales, compuesta de lecho rocoso metamórfico y plutónico del Paleozoico Inferior bajo rocas sedimentarias Cenozoicas principalmente de origen volcánico (Seggiaro et al. 2014). El río Hualfín es un importante agente geomorfológico que atraviesa varias terrazas aluviales cuaternarias. La mayoría de los sitios arqueológicos conocidos en esta área se localizan sobre y/o alrededor de estas terrazas.

El sitio Villavil 1 (Figura 1) se encuentra al norte del valle, sobre una meseta aluvial inmediata al río homónimo, a una altura de 1850 m s.n.m. El mismo está dividido en dos sectores, A y B respectivamente, donde se han identificado varias estructuras de roca rectangulares, cuadrangulares y sub-rectangulares, cerradas (recintos) y abiertas (Figura 2c), posiblemente definiendo sectores vinculados a la actividad agrícola. Los fechados obtenidos hasta el momento permiten relacionar las ocupaciones del sitio desde el período Formativo Temprano-Medio (ca. 200 a.C - 900 d.C.) al de Desarrollos Regionales/Inka (ca. 900 - 1430 d.C.) (Lynch 2014; Lynch y Giovannetti 2018). A partir de los fragmentos cerámicos recuperados en superficie se identificaron estilos (Ciénaga/Aguada, Belén y Santamariano) que apoyan esta cronología, aunque se registró una predominancia de cerámica correspondiente al período Formativo Medio (Lynch et al. 2020). 


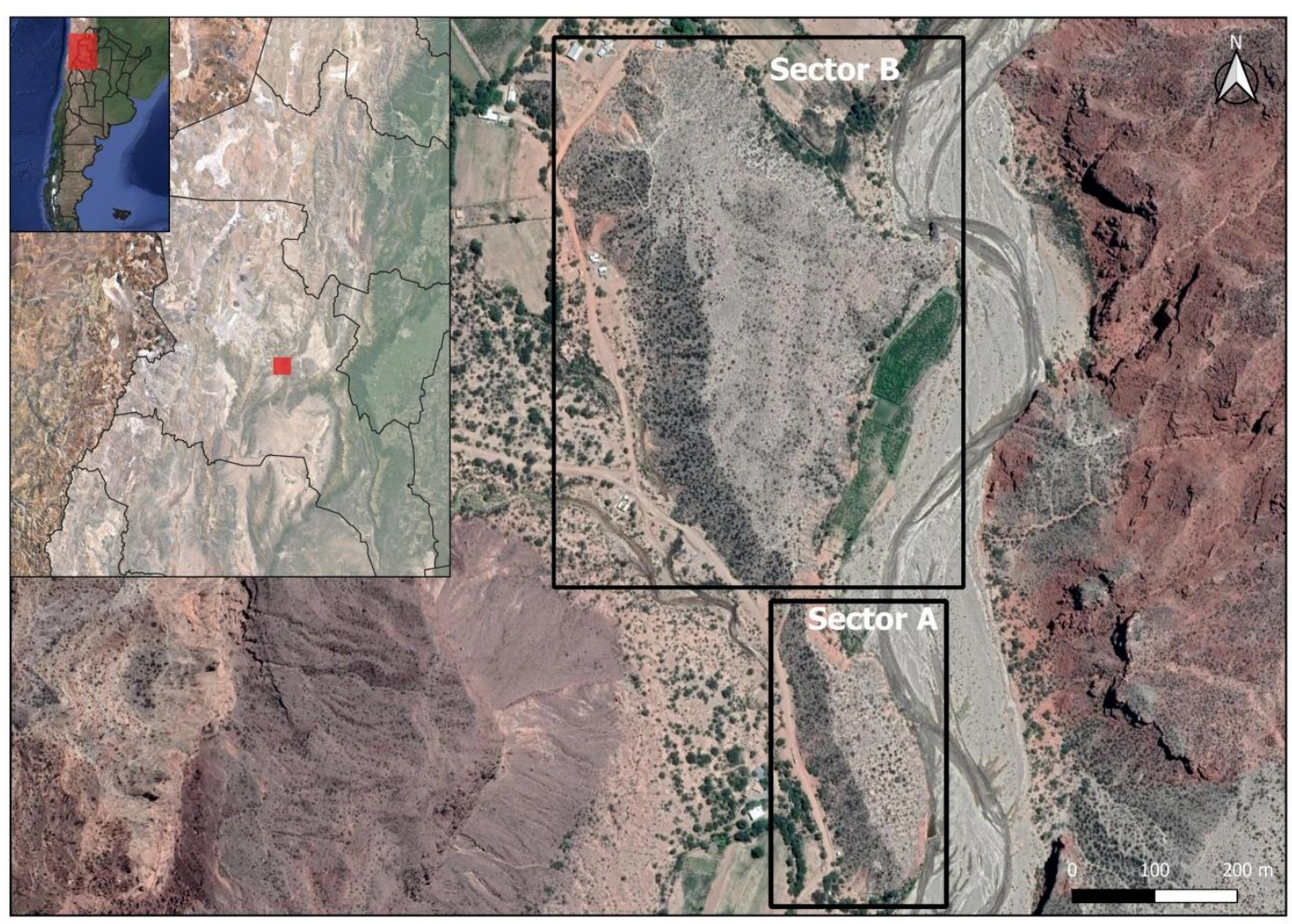

Figura 1. Ubicación de los Sectores A y B del sitio Villavil 1.

\section{Materiales y métodos}

Los materiales analizados en este trabajo fueron recuperados durante la campaña arqueológica que se desarrolló en el 2017 en el sitio. El trabajo de campo consistió en un muestreo dirigido, con cuadrículas de $25 \mathrm{~m}^{2}$, en donde se seleccionaron puntos de recolección superficial de 1,5 o 3 m de diámetro, teniendo en cuenta la visibilidad y estabilidad del terreno. Se plantearon 46 puntos de recolección (Figura 2a-b), 23 en el sector A y 23 en el sector B (Lynch et al. 2019; Lynch et al. 2020). En este muestreo se recolectó material lítico, así como cerámico que se encuentra actualmente bajo estudio.

La muestra lítica recuperada está compuesta por un total de 234 piezas, que para su análisis fueron segmentadas en diferentes clases tipológicas (Aschero 1975; Aschero y Hocsman 2004): artefactos formatizados (AF), artefactos no formatizados (ANF), desechos de talla (DT), núcleos y artefactos no modificados por talla. En Bentivenga y Ríos Malan (2020) se presentó el análisis preliminar del conjunto y, en esta oportunidad, se desarrolla el estudio de palas y/o azadas incluidas en el conjunto de artefactos formatizados (ver Tabla 1). 


\section{VILLAVIL 1}

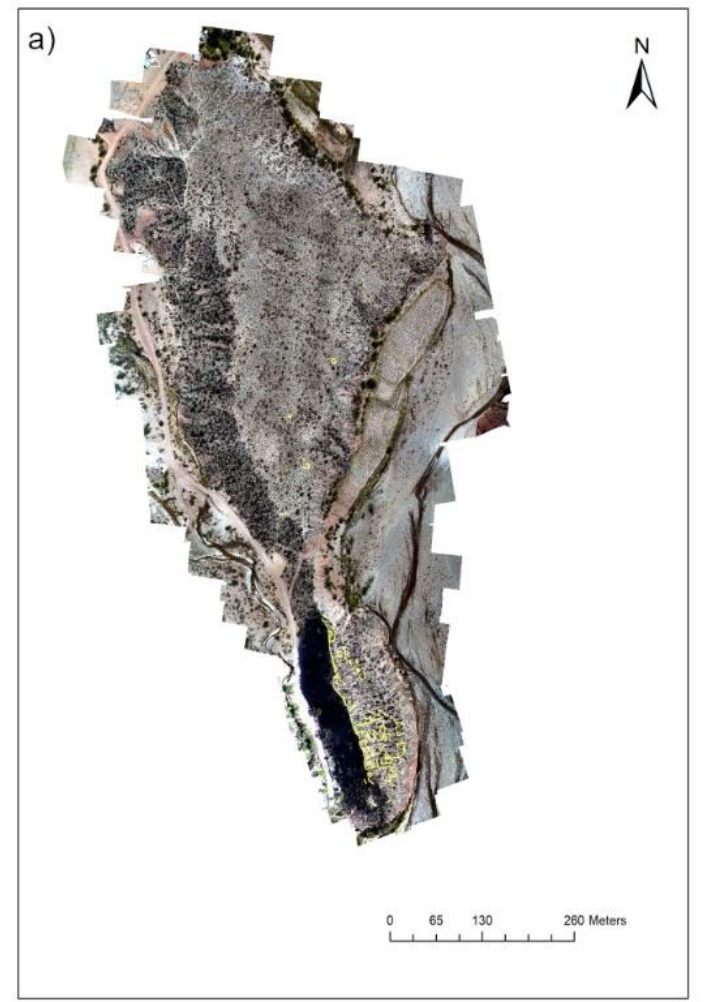

b)

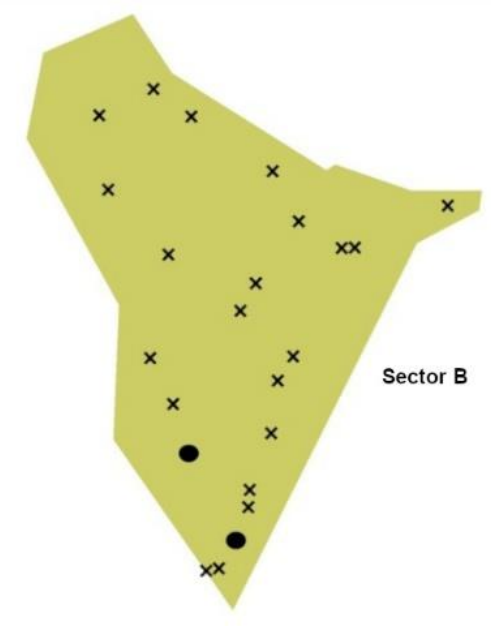

c)

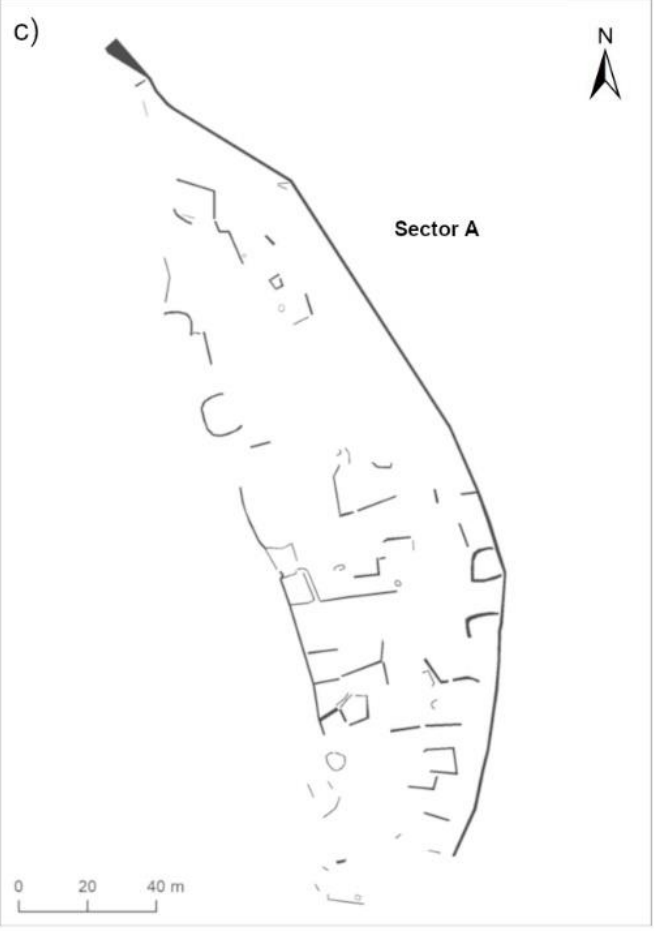

Figura 2. a) Ortoimagen del sitio Villavil 1. b) Distribución de las áreas de recolección en los sectores A y B, respectivamente. Los puntos hacen referencia a áreas donde se recuperaron palas y/o azadas. c) Plano del Sector A. 


\begin{tabular}{lcccc}
\hline \multirow{2}{*}{ Clases Tipológicas } & \multicolumn{2}{c}{ Villavil 1 (Sector A) } & \multicolumn{2}{c}{ Villavil 1 (Sector B) } \\
\cline { 3 - 5 } & $\mathbf{N}$ & $\mathbf{0}$ & $\mathbf{n}$ & $\%$ \\
\cline { 3 - 5 } AF (Cepillo) & 1 & 0,71 & 0 & 0,00 \\
AF (Palas / Azadas) & 4 & 2,86 & 2 & 2,13 \\
DT & 106 & 75,71 & 78 & 82,98 \\
Núcleos & 6 & 4,29 & 1 & 1,06 \\
No Modificado por Talla & 18 & 12,86 & 13 & 13,83 \\
TOTAL & 5 & 3,57 & 0 & 0,00 \\
\hline
\end{tabular}

Tabla 1. Frecuencias absolutas y porcentaje de las Clases Tipológicas halladas en Villavil 1: artefactos formatizados (AF), artefactos no formatizados (ANF), desechos de talla (DT), núcleos y artefactos no modificados por talla. Las palas y/o azadas fueron segregadas de los otros grupos de artefactos formatizados, en función de los objetivos de este trabajo.

Teniendo en cuenta los conceptos teóricos considerados previamente, en este trabajo se llevó a cabo la descripción tecno-morfológica de los materiales siguiendo las propuestas de Aschero $(1975,1983)$ y Pérez (2012). A partir de esto se busca determinar ciertas tendencias en el modo de producción de estos artefactos; así como identificar el nivel de inversión de trabajo a través de los lineamientos de Hocsman y Escola (2006-2007).

Las variables consideradas para este análisis fueron: materia prima, estado de la pieza (entero o fracturado), presencia de pedúnculo, morfología general del cuerpo (circular, triangular, sub-circular, con o sin pedúnculo) (Pérez, 2012); forma base, tamaño (dimensiones absolutas de largo, ancho y espesor máximos), módulo, peso, porcentaje de remanente de corteza, serie técnica (ancho y extensión de los lascados sobre las caras, y situación de los lascados respecto a las caras de las piezas) de cada uno de los bordes (identificados según la cuadrícula de localización), conformación del borde o superficie, regularidad del borde y la arista activa, estado del filo activo y ángulo del filo (medido o estimado) (Aschero 1975, 1983).

En cuanto a la identificación de las materias primas, se realizaron estudios macroscópicos y bajo lupa binocular (Nikon SMZ 745 a una magnificación de 50X), considerando las variables textura (tamaño y variación granulométrica), color y tipo de fractura.

En relación con el análisis del nivel de trabajo invertido en la manufactura, Hocsman y Escola (2006-2007) proponen utilizar cuatro categorías: clase técnica, requerimiento de extracción, requerimiento de formatización de la forma base e imposición de forma. 
La clase técnica permite medir la inversión de trabajo de percusión o presión en función del grado en que se ven afectadas una o ambas caras de la pieza. En consecuencia, el adelgazamiento o la reducción requieren un mayor esfuerzo que el lascado no invasivo, así como también mayores conocimientos y destreza técnica.

Con respecto al diseño, la variable requerimientos de extracción de la forma base hace referencia a la búsqueda o no de un soporte con cierta morfología y tamaño específico para su posterior formatización. En función de esto, se discrimina entre percusión dirigida o no dirigida. La primera implica mayor inversión de trabajo, dado que incluye aquellos casos donde se busca manufacturar un tipo particular de instrumento, lo que exige obtener formas bases con atributos morfológicos y dimensionales muy específicos. Por su parte, la percusión no dirigida se corresponde con artefactos cuya manufactura no demanda una elección tan exhaustiva de formas bases, pudiéndose utilizar una amplia gama de soportes que respondan a mínimos requerimientos.

Entre las otras dimensiones de diseño, los requerimientos de formatización de la forma base hacen referencia a la presencia o ausencia de rasgos particulares en los soportes que impliquen un esfuerzo de producción, como puede ser la necesidad de generar una arista sobre un clasto. En tanto, mediante la variable requerimientos de imposición de forma se evalúa el esfuerzo invertido, por ejemplo, en diferenciar determinadas partes de los artefactos o en realizar ciertas morfologías específicas de lascado sobre sus caras; ciertos artefactos, por las características de su diseño, involucran una mayor inversión de trabajo.

\section{Resultados}

El conjunto analizado consiste en seis ejemplares, de los cuales tres se encuentran enteros y el resto fracturados. A continuación, se describen las características de las materias primas a partir de las cuales fueron manufacturadas, así como las variables tecno-morfológicas seleccionadas.

Las materias primas utilizadas fueron reconocidas como granito y basalto. La primera es de coloración gris claro, con textura fanerítica de grano grueso (mayor a 5 $\mathrm{mm}$ ) y fractura irregular, mientras que el basalto fue definido por su color gris oscuro, su textura afanítica y fractura concoidea.

Pieza Nº 1 (VV1-S107-32):

La pieza $N^{o} 1$ (Figuras 3 y 4) se encuentra entera y confeccionada sobre un lascón de granito con remanente de corteza por encima del $50 \%$. Las dimensiones absolutas máximas son $270 \mathrm{~mm}$ de largo, $185 \mathrm{~mm}$ de ancho y $31 \mathrm{~mm}$ de espesor, módulo mediano normal, mientras que el peso es de $1895 \mathrm{~g}$. El espesor del pedúnculo es de 23,5 $\mathrm{mm}$, el de las inflexiones del cuerpo es de $11 \mathrm{~mm}$ y 21,5 mm, y el del cuerpo de 4,4 mm. El borde del cuerpo (A34) presenta filo natural, regular y embotado astillado con un 
ángulo estimado de $30^{\circ}$; mientras que cada una de las dos inflexiones del cuerpopedúnculo fueron formatizadas por lascado simple unifacial extendido (B5) y parcialmente extendido (B6), respectivamente, junto con retoques y microrretoques en ambas caras de la pieza (Figura 3a). El borde del pedúnculo (B8UW) muestra retalla marginal unifacial con un filo embotado astillado. La morfología general de la pieza fue clasificada como elíptica regular con pedúnculo esbozado (Figura 3a-b y 4).

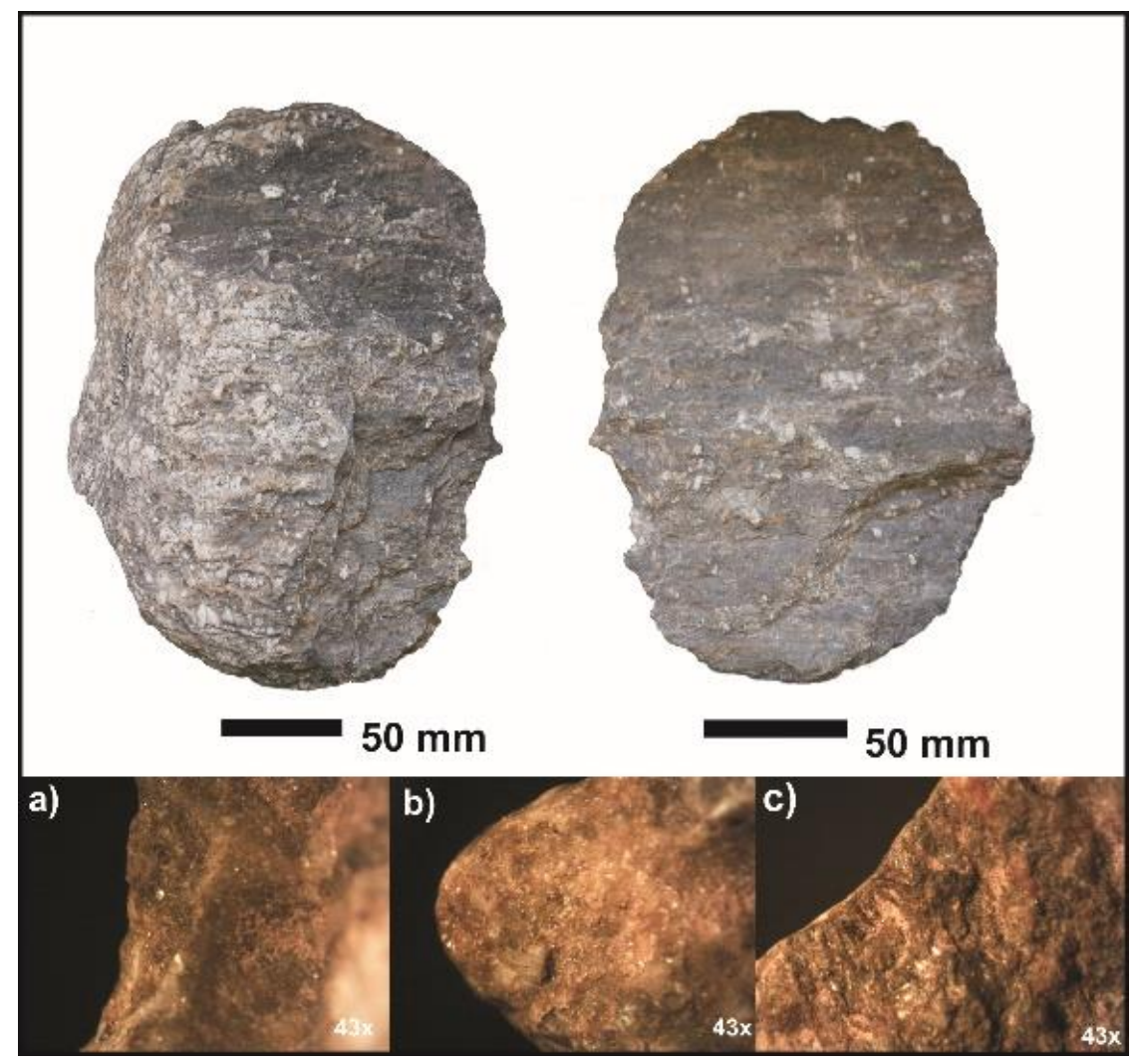

Figura 3. Pieza $\mathrm{N}^{\circ} 1$ (VV1-S107-32). Arriba: vista general de caras dorsal y ventral. Abajo: a) Retoques y microrretoques escalonados en la porción medial y proximal (área de enmangue) de la pieza. b) Detalle a mayor aumento del área de curvatura en el sector de enmangue. c) Redondeamiento y estrías oblicuas en borde lateral de la pieza.

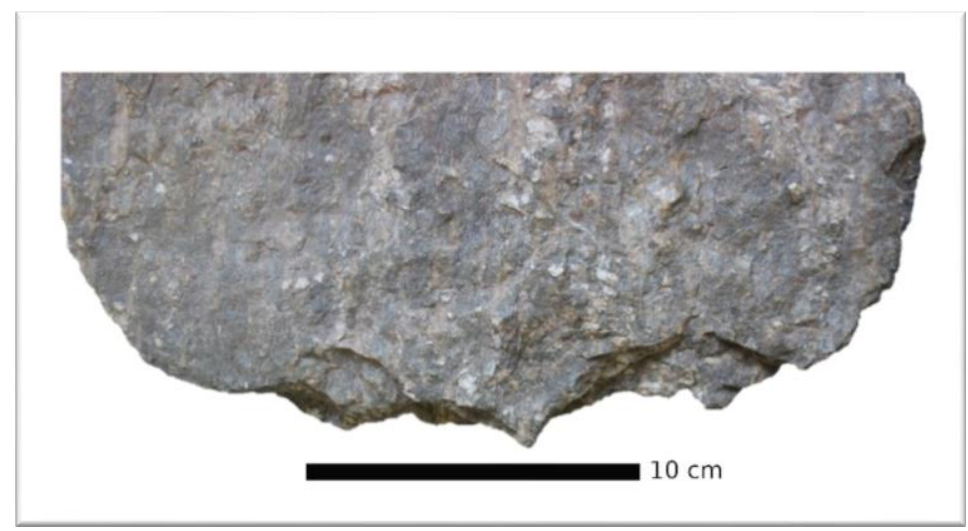

Figura 4. Pieza No1 (VV1-S107-32). Vista en detalle del borde en cara ventral. 
Pieza No2 (VV1-S103-7):

El ejemplar №2 (Figura 5), en estado entero, está confeccionado sobre una lasca primaria de basalto que conserva la totalidad de la corteza. Su longitud máxima es de $185 \mathrm{~mm}$, mientras que el ancho máximo es de $110 \mathrm{~mm}$ y el espesor máximo de $28 \mathrm{~mm}$, de módulo mediano alargado. Su peso es de $834 \mathrm{~g}$. El sector distal (A17W), con un espesor de $6 \mathrm{~mm}$, está formatizado por retoque y microrretoque marginal unifacial (Figura 5a), conformando un filo normal con espolones, regular y activo con astilladuras múltiples, y un ángulo de $64^{\circ}$. El borde B31 del cuerpo presenta un espesor de $5 \mathrm{~mm}$ y corresponde a un filo natural regular y activo con un ángulo de $58^{\circ}$, mientras que el espesor del borde B24 es de $7 \mathrm{~mm}$ y está formatizado por retoque marginal unifacial, presentando un filo natural regular y activo con astilladuras múltiples y un ángulo de $55^{\circ}$. En el caso de las inflexiones del cuerpo, el borde A53 está formatizado por retoque marginal unifacial y se encuentra festoneado, con un filo regular y activo con astilladuras múltiples, y un ángulo estimado de $74^{\circ}$; mientras que el borde A46 no presenta formatización y corresponde a un filo natural, regular y activo de 57\%; los espesores son de 8 y $6 \mathrm{~mm}$ respectivamente. La base (A8UW), de 27 $\mathrm{mm}$ de espesor, no muestra formatización. La forma de la pieza es elíptica regular con pedúnculo esbozado.

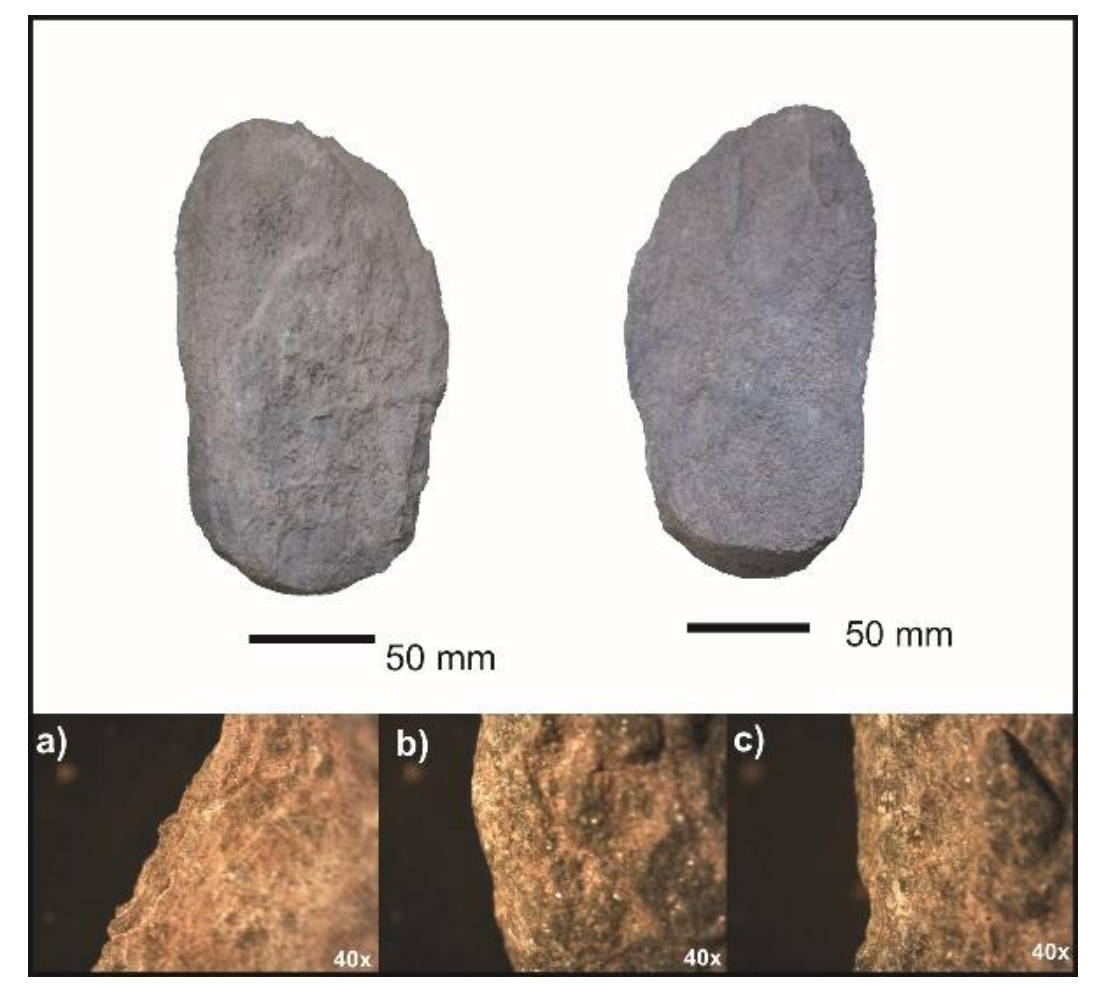

Figura 5. Pieza No2 (VV1-S103-7): Arriba: vista general de caras dorsal y ventral. Abajo: a) Retoques y microrretoques en el borde de la porción distal. b-c) redondeamiento y estrías longitudinales en el borde distal de la pieza. 
Pieza Nº 3 (VV1-S127-172):

La pieza No3 (Figura 6a) se encuentra entera y realizada sobre una lasca secundaria de basalto que preserva corteza en más de un $50 \%$. Sus dimensiones máximas de largo, ancho y espesor son 120,5 mm, 120,7 mm y $28 \mathrm{~mm}$, respectivamente, de módulo corto ancho; mientras que el peso es de $430 \mathrm{~g}$. El pedúnculo tiene un espesor de $26 \mathrm{~mm}$, mientras que los bordes del cuerpo y el sector distal varían entre los 3 y $6 \mathrm{~mm}$ de espesor. El sector distal (A7UW) presenta filo natural, regular y activo con astilladuras múltiples, y un ángulo de $49^{\circ}$, aunque al igual que el pedúnculo -con filo natural activo (A8UW) - no está formatizado. Los dos bordes del cuerpo presentan retoques marginales unifaciales, conformando filos normales, regulares y activos, con un ángulo medido de $67^{\circ}$ (A51) y estimado de $44^{\circ}$ (A24), respectivamente. Se incluyó dentro de la morfología triangular corta con pedúnculo.

Pieza Nº 4 (VV1-S109-25):

Fue identificada como una lasca indiferenciada de granito con reserva de corteza en más del $50 \%$ de la cara dorsal (Figura 6b). Se encuentra fracturada y se reconoció como parte de una base. Presenta 96,6 $\mathrm{mm}$ de largo, $72 \mathrm{~mm}$ de ancho y $22 \mathrm{~mm}$ de espesor máximos, de módulo mediano normal y con un peso de 196 g. Su borde (A56), sin formatización, constituye un filo natural, regular y embotado astillado, con un ángulo de $84^{\circ}$. No fue posible identificar la morfología general de la pieza.

Pieza N 5 (VV1-S117-140):

Esta pieza se encuentra fracturada y fue confeccionada sobre lasca indiferenciada de basalto, con presencia total de corteza en la cara dorsal (Figura 6c). Se identificó solo una arista (A51) correspondiente a un filo natural, regular y activo, con un ángulo estimado de $44^{\circ}$. La longitud máxima es de $96,1 \mathrm{~mm}$, con $82,1 \mathrm{~mm}$ de ancho y 31,2 $\mathrm{mm}$ de espesor máximos y módulo mediano normal. El peso es de $226 \mathrm{~g}$. El estado de fragmentación no permitió reconocer la forma de la pieza.

Pieza Nº (VV1-S136-245):

Es una lasca secundaria fracturada extraída de una laja basáltica (Figura 6d). Fue identificada como un borde distal que conserva más del $50 \%$ de remanente de corteza. Sus dimensiones máximas son 120,93 mm de largo, $73 \mathrm{~mm}$ de ancho y $11 \mathrm{~mm}$ de espesor, de módulo mediano alargado y un peso de 155 g. Presenta un borde formatizado (A56) por retoque marginal unifacial con filo festoneado, regular y activo, con un ángulo de $49^{\circ}$. No se logró identificar la morfología de la pieza. 
a)

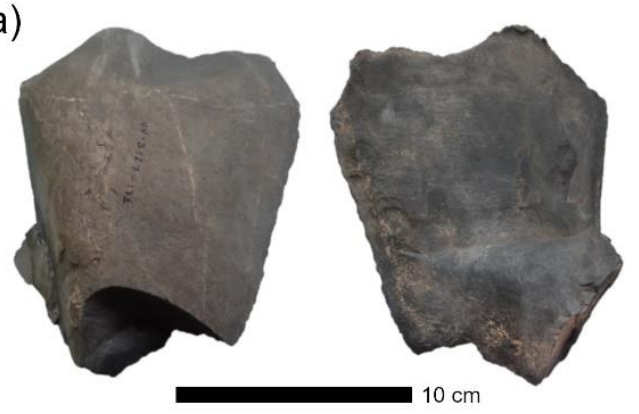

c)

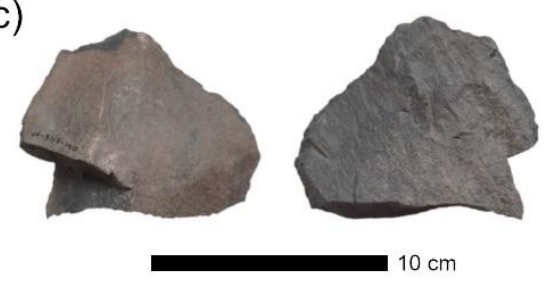

b)

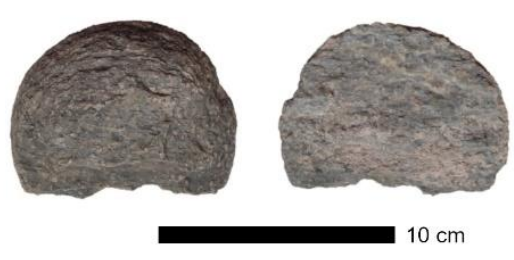

d)

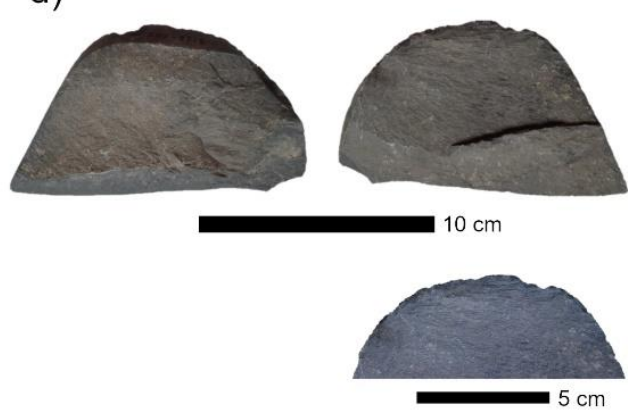

Figura 6. Palas/azadas recuperadas en Villavil 1, vista de caras dorsal y ventral. a) Pieza $\mathrm{N}^{\circ} 3$ (VV1-S127-172). b) Pieza N 4 (VV1-S109-25). c) Pieza N 5 (VV1-S117-140). d) Pieza No 6 (VV1S136-245) y mayor detalle del borde de la cara ventral.

A partir de los análisis realizados, se observó una importante variabilidad en lo que respecta a la conformación, regularidad y estado de los filos, a las series técnicas empleadas, a las dimensiones absolutas y al módulo de las piezas enteras, que varía entre mediano-largo y corto-ancho. La mayoría de los filos son naturales, sin embargo, existen algunos ejemplares en donde se realizó una formatización unifacial previa que generó una forma primaria festoneada. Asimismo, la formatización, mayormente unifacial, más frecuente corresponde al retoque marginal, aunque en menor medida también se registró retalla y lascado simple. La utilización de la técnica de pulido no fue registrada en ninguno de los ejemplares analizados. Es notorio en todas las piezas enteras que el espesor de los pedúnculos es mayor al de las inflexiones, y el grosor de éstas sobrepasa al de los cuerpos.

A su vez, la predominancia de clases técnicas de trabajo no invasivo unifacial (retoque y retalla marginales), así como de filos naturales sin formatización, indican un bajo costo tecnológico. Sin embargo, los aspectos de diseño, sí muestran un mayor grado de trabajo y destreza técnica. La utilización de lascas de gran tamaño indica requerimientos de extracción de la forma-base por percusión dirigida, además se aprecian requerimientos de formatización de los bordes o aristas de la forma-base e imposición de forma (sector distal, cuerpo y pedúnculo). 


\section{Discusión}

Los resultados alcanzados hasta el momento, a partir de esta primera aproximación al conjunto de palas y/o azadas procedentes del sitio Villavil 1, permitieron caracterizarlas tecno-morfológicamente.

En cuanto al uso de materias primas, la mayoría de los antecedentes registran una preferencia por la utilización de esquistos, pizarras o filitas para la confección de estos instrumentos (Avalos 1998; Borgo 2014; Fabron 2012; Giovannetti 2009; Leoni et al. 2014), aunque también se habrían seleccionado otras rocas de alta dureza tales como basalto-andesita con diaclasamiento, de apariencia o soporte lajoso (Avalos 1998; Gastaldi 2008; Pérez 2010, 2012). Las piezas consideradas en este trabajo permiten observar la selección de basalto y rocas graníticas para la producción de palas y/o azadas, a pesar de que en la zona se encuentran disponibles otras rocas metamórficas tales como esquistos y pizarras/filitas (Lynch et al. 2016; Seggiaro et al. 2014). Las rocas empleadas presentan un alto grado de dureza y distintas calidades para la talla (Aragón y Franco 1997) que, sumado a su amplia disponibilidad en el área, habrían favorecido su selección acorde con la funcionalidad a la que habrían sido destinadas (remover la tierra). Sin embargo, llama la atención la ausencia en el registro de la utilización de cuarzo y/o andesita, materias primas que se encuentran representadas en el resto del conjunto lítico de Villavil 1 e igualmente disponibles a nivel local (Bentivenga y Ríos Malan 2020; Lynch et al. 2016).

En este sentido y a pesar del tamaño reducido de la muestra, se puede proponer que el granito y el basalto habrían sido seleccionados para la confección de palas y/o azadas en detrimento de otras materias primas disponibles. Esta selección podría estar relacionada con la existencia de grandes nódulos que permitieran la obtención de formas base de tamaño grande a muy grande (sensu Aschero 1975), indispensables para un volumen acorde a las necesidades funcionales de este conjunto.

La mayoría de los bordes de la sección activa de las piezas enteras se encuentran formatizados por lascados unifaciales, y los filos de las piezas No2 (Figura 5) y No3 (Figura 6a) se hallan activos. A su vez, las inflexiones y aletas del cuerpo de la pieza $\mathrm{N}^{\circ} 1$ (Figura 3 y 4) y una de las inflexiones de la pieza $\mathrm{N}^{\circ} 2$ se encuentran formatizadas, lo cual estaría indicando la intención de obtener formas que puedan ser enmangada, y en ambos casos el pedúnculo se encuentra embotado. La pieza No3, en cambio, presenta un pedúnculo natural marcado que no habría requerido tareas de formatización.

Por otra parte, el sector distal y los bordes del cuerpo de las piezas enteras son más delgados, mientras que los pedúnculos y las inflexiones del cuerpo-pedúnculo presentan un mayor espesor, acorde con lo descripto por Pérez $(2010,2012)$ y asociado a la obtención de un refuerzo para evitar la fractura durante su utilización. 
Respecto a la inversión de trabajo requerido para la confección de las palas y/o azadas, la propuesta de Hocsman y Escola (2006-2007) permite observar cierta complejidad. Por un lado, se puede preliminarmente señalar que la clase técnica unifacial no invasiva estaría indicando un bajo esfuerzo de formatización. Sin embargo, por otro lado, los diseños de las palas/azadas analizadas habrían conllevado cierta inversión de trabajo y destreza técnica, considerando el requerimiento de obtener lascas de gran tamaño, y la búsqueda de confeccionar formas determinadas y específicas, con pedúnculo más o menos esbozado. A su vez, se debe tener en cuenta que estos artefactos son elementos compuestos, es decir, necesitan de la producción de un enmangue y diferentes tipos de ataduras que permitan un correcto agarre y uso de las mismas. De lo contrario, las fracturas o deslizamientos serían recurrentes e imposibilitarían su utilización.

De esta manera, las dimensiones de las lascas y la búsqueda intensional de formas con los requerimientos necesarios para unir a un mango involucran una anticipación al momento de tallar las hojas. Esta situación las volvería excepcionales dentro del conjunto lítico de Villavil 1, donde la estrategia tecnológica es predominantemente expeditiva y el número de artefactos formatizados hallados hasta el momento es escaso (Bentivenga y Ríos Malan 2020; Lynch y Lynch 2016).

Si bien las piezas procedentes de la Puna jujeña y catamarqueña que analizó Pérez $(2010,2012)$ requirieron una mayor formatización y son artefactos bien estandarizados, los ejemplares de Villavil 1 fueron confeccionados sobre materias primas locales, y presentan tanto rasgos como morfologías que posibilitarían su enmangue y uso como palas o azadas sin un alto grado de formatización.

\section{Consideraciones finales}

Las características de Villavil 1 indican que probablemente haya sido un sitio donde predominaron las actividades de índole agrícola. Es posible que en el mismo se manifiesten aspectos que estuvieran vinculados con la vida aldeana de quienes fueran los trabajadores en los campos de cultivo del área (Lynch y Giovannetti 2018). Las palas y/o azadas identificadas se registraron cercanas a lugares habitacionales, como así también a canales de riego. Es por ello que estas herramientas pudieron haber jugado un rol fundamental en las tareas de producción de alimento, excavación y mantenimiento de canales, remoción de malezas o limpieza de parcelas, entre otras.

El análisis del resto de los materiales recuperados del sitio nos permite plantear que los habitantes de esta región utilizaron estrategias de tecnología lítica principalmente expeditivas (Bentivenga y Ríos Malan 2020; Lynch y Lynch 2016). No obstante, el conjunto de palas y/o azadas correspondería a un modo más conservativo debido a que su producción requirió un mayor tiempo de trabajo y esfuerzo. 
Si bien hasta el momento el conjunto analizado presenta un número reducido de ejemplares y el alcance del estudio no permite aseverar el uso de estos artefactos sobre diferentes materiales, a futuro se considera realizar estudios traceológicos (identificación concreta de los modos de uso y materiales) como así también análisis de micro-residuos para la detección de especies vegetales, que permitan relacionar a nuevos ejemplares de este grupo procedentes de contexto estratigráfico con diferentes actividades de laboreo de la tierra. A pesar de esto, el trabajo presentado es una primera aproximación a la caracterización de posibles palas y/o azadas líticas procedentes del área bajo estudio, y en este sentido implica un avance en el registro y análisis de un diseño instrumental que, hasta el momento, no había sido descripto. Por último, creemos que la estrategia de mayor conservación implicada en la manufactura de dichos artefactos demuestra la importancia de las prácticas agrícolas para las poblaciones locales en diferentes momentos de ocupación del valle.

Agradecimientos: Queremos agradecer al CONICET por el financiamiento (PIP Nº11220170100033), así como a Milagros Ríos Malan, César Parcero-Oubiña y Pastor Fábrega-Álvarez por su participación y asistencia metodológica en el trabajo de campo. También extendemos nuestro agradecimiento al Comité Editorial y a los evaluadores anónimos por sus valiosos comentarios que permitieron mejorar notablemente la calidad del trabajo. Todo lo escrito en este trabajo es entera responsabilidad de los autores.

\section{Bibliografía citada}

Alfaro de Lanzone, L.C.

1969 Exploraciones arqueológicas en la Puna de Jujuy. Antiquitas, 8: 7-13.

Álvarez, M.R.

2004 Producción lítica en el pukara de Tilcara. Mosaico. Trabajos en Antropología Social y Arqueología, 1: 25-34.

Andrefsky, W.

1994 Raw-Material Availability and the Organization of Technology. American Antiquity, 59(1): 21-34. https://doi.org/10.2307/3085499

Aragón, E. y N.V. Franco

1997 Características de rocas para la talla por percusión y propiedades petrográficas. Anales del Instituto de la Patagonia, 25: 187-199.

Aschero, C.

1975 Ensayo para una clasificación morfológica de artefactos líticos aplicada a estudios tipológicos comparativos. Informe al CONICET. Ms. Buenos Aires. 
1983 Ensayo para una clasificación morfológica de artefactos líticos aplicada a estudios tipológicos comparativos. Apéndices A-C. Informe al CONICET. Ms. Buenos Aires.

Aschero, C. y S. Hocsman

2004 Revisando cuestiones tipológicas en torno a la clasificación de artefactos bifaciales. Temas de arqueología. Análisis lítico, 7-25.

Avalos, J.C.

1998 Modos de uso de implementos agrícolas de la Quebrada de Humahuaca y Puna a través del análisis de huellas de desgaste. Los desarrollos locales y sus territorios. Arqueología del NOA y sur de Bolivia (comp. por B. Cremonte), pp. 285-303. Universidad Nacional de Jujuy, S. Salvador de Jujuy.

2003 Sistemas de producción lítica de las sociedades tardías de la Quebrada de Humahuaca. Cuadernos de la Facultad de Humanidades y Ciencias Sociales - Universidad Nacional de Jujuy, 20: 271-290.

Bennett, W.C.

1948 The North. Northwest Argentine Archeology (ed. por W. C. Bennett, E. F. Bleiler y F. H. Sommer), pp. 19-43. Department of Anthropology, Yale University Publication in Anthropology, 38, New Haven.

Bentivenga, E. y M. Ríos Malan

2020 Aproximación a la Producción Lítica de los sitios Villavil 1 y Villavil 2, Hualfín, Catamarca. Cuadernos del Instituto Nacional de Antropología y Pensamiento Latinoamericano - Series Especiales, 8(1): 47-59.

Bogaard, A.

2005 'Garden agriculture' and the nature of early farming in Europe and the Near East. World Archaeology, 37(2): 177-196. https:// doi.org/10.1080/00438240500094572

Borgo, M.

2014 Primeros estudios sobre tecnología lítica en sitios del Período de Integración Regional en la provincia de La Rioja. El caso de la Cuestecilla. La zaranda de ideas. Revista de Jóvenes Investigadores en Arqueología, 11: 47-66.

Capparelli, A., S.M. Valamoti y M.M. Wollstonecroft

2011 After the harvest: Investigating the role of food processing in past human societies. Archaeological and Anthropological Sciences, 3(1): 1-5. https://doi.org/10.1007/s12520-011-0063-2

Carbonelli, J.P. y E.G. Gaál

2015 La tecnología lítica de las ocupaciones formativas durante el primer milenio de la era en el sur Yocavil y áreas aledañas al valle (pcia. de Catamarca). Cuadernos del Instituto Nacional de Antropología y Pensamiento Latinoamericano - Series Especiales, 2(2): 30-52. http://ppct.caicyt.gov.ar/index.php/cinapl-se/article/view/7507 
Chaparro, M.G.

2008-2009 La tecnología en Tolombón: Nuevas contribuciones al estudio de las sociedades tardías del NOA. Anales de Arqueología y Etnología, 63-64: 107-136.

Childe, V.G.

1998 [1936]. The Dawn of European Civilization. Routledge, New York.

Clemente, I. y J.F Gibaja.

1998 Working Processes on Cereals: An Approach Through Microwear Analysis. Journal of Archaeological Science, 25(5): 457-464. https://doi.org/10.1006/jasc.1997.0214

Elías, A.M.

2012 Tecnología lítica en el periodo tardío (ca. 1100-550 años AP) de Antofagasta de la Sierra (Provincia de Catamarca, Puna Meridional Argentina). Relaciones de la Sociedad Argentina de Antropología, 37(1): 19-41.

Escola, P.S.

2000 Tecnología lítica y sociedades agropastoriles tempranas. Tesis Doctoral Inédita. Universidad de Buenos Aires, Buenos Aires.

2004 La expeditividad y el registro arqueológico. Chungará (Arica), 36: 49-60. https://doi.org/10.4067/S0717-73562004000300008

Fabron, G.

2012 Análisis de tecnología lítica: Palas y/o azadas. Antumpa, un caso de estudio (pcia. de Jujuy). Anuario de Arqueología, 4: 205-218.

Fairbairn, A.

2005 A history of agricultural production at Neolithic Çatalhöyük East, Turkey. World Archaeology, 37(2): 197-210. https:// doi.org/10.1080/00438240500094762

Flores, M.C.

2012 El cuarzo como materia prima en el valle de Hualfín (provincia de Catamarca, Argentina) durante el Período de Desarrollo Regionales/Inka. Comechingonia, 16(1): 297-305.

2014 Uso de rocas sedimentarias en el valle de Hualfín prehispánico. Comechingonia, 18(2): 263-271.

Flores, M.C., y F. Wynveldt

2009 Análisis tecno-tipológico de los artefactos líticos de la Loma de los Antiguos de Azampay (Departamento de Belén, Catamarca). Intersecciones en antropología, 10(1): 221235. 
Gaál, E.G.

2011a Un acercamiento preliminar al estudio de la base local de recursos líticos y al análisis artefactual en el poblado arqueológico de Rincón Chico (período Tardío), valle de Yocavil, Catamarca. Comechingonia Virtual: Revista Electrónica de Arqueología, 5(1): 138 .

2011b Base regional de recursos líticos y fuentes potenciales de aprovisionamiento en el sur del valle de Yocavil, Catamarca. Arqueogasta. Estudiando el pasado... repensando el futuro (comp. por A. Calisaya, V. Erramouspe y V.B. Martin Silva), pp. 70-73. Edición AD Calisaya, S. Miguel de Tucumán.

Gastaldi, M.R.

2008 La Pala: Medio de Producción del Modo de Producción. Problemáticas de la Arqueología Contemporánea. Publicaciones del XV Congreso Nacional de Arqueología Argentina (comp. por A. Austral y M. Tamagnini), pp. 385-399. Universidad Nacional de Río Cuarto, Río Cuarto.

Giovannetti, M.A.

2009 Articulación entre el sistema agrícola, redes de interacción y áreas de molienda como medida del grado de ocupación Inka en El Shincal y Los Colorados (Prov. de Catamarca). Tesis Doctoral Inédita. Universidad Nacional de La Plata, La Plata.

González, A.R.

1955 Contextos culturales y cronología relativa en el área central del NO argentino. Anales de arqueología y etnología, 11: 7-32.

1979 Dinámica cultural del NO argentino. Evolución e historia en las culturas del NO argentino. Antiquitas, 28-29: 1-15.

Haber, A.F. y Gastaldi, M.G.

2006 Vida con palas. Antípoda, 2: 275-302.

Hocsman, S. y P.S. Escola

2006-2007 Inversión de trabajo y diseño en contextos líticos agro-pastoriles (Antofagasta de la Sierra, Catamarca). Cuadernos del INAPL, 21: 75-90.

Kolata, A.L.

1985 El papel de la agricultura intensiva en la economía política del estado de Tiwanaku. Diálogo Andino, 4: 11-38.

Kvietok, D.P.

1987 Digging sticks or daggerboards? A functional analysis of wooden boards from the Ica region. Andean Past, 1(1): 247-274. 
Leoni, J.B., G. Fabron, D.S. Tamburini, A. Hernández y C.G. Brancatelli

2014 «Cóndor 2», un sitio del período de Desarrollos Regionales 1 en el sector norte de la quebrada de Humahuaca, Jujuy. Estudios Sociales del Noa: nueva serie, 13: 125-146.

Lynch, J.

2014 Sitio Villavil: Una aproximación a la dinámica local- estatal al norte del valle de Hualfín, Catamarca. Intersecciones en Antropología, 15: 24-32.

Lynch, J. y V. Lynch

2016 Análisis de la tecnología lítica del sitio Villavil localizado en el valle de Hualfín (Provincia de Catamarca, Argentina). Revista Española de Antropología Americana, 46: 265-282. https:// doi.org/10.5209/REAA.58297

Lynch, J., V. Lynch y E. Villalba

2016 Estudios petrográficos y de cortes delgados para la identificación de materias primas líticas en el sector norte del valle de Hualfín (Catamarca, Argentina). Arqueologia Iberoamericana, 30: 70-76. https://doi.org/10.5281/zenodo.1317029

Lynch, J. y M.A. Giovannetti

2018 Paisajes Inka en el centro-oeste de Catamarca (Argentina). Latin American Antiquity, 29(4): 754-773. https://doi.org/10.1017/laq.2018.50

Lynch, J., C. Parcero-Oubiña y P. Fábrega-Álvarez

2020 A field experience of documentation and analysis through 3D mapping and surface survey in the Hualfín Valley (Catamarca, Argentina). Digital Applications in Archaeology and Cultural Heritage, 17: 1-11. https:// doi.org/10.1016/j.daach.2020.e00145

Lynch, J., C. Parcero-Oubiña, P. Fábrega-Álvarez, E. Bentivenga, M. Ríos Malan y L. Blanco

2019 Uso diferencial del paisaje: Nuevos acercamientos metodológicos al Norte del valle de Hualfín, Catamarca. Libro de Resúmenes del XX Congreso Nacional de Arqueología Argentina (comp. por A. Laguens, M. Bonnin y B. Marconetto). Universidad Nacional de Córdoba, Córdoba. https:// digital.csic.es/handle/10261/209652

Márquez Miranda, F.

1939 Cuatro viajes de estudio al más remoto Noroeste Argentino. Revista Museo de La Plata, Antropología, 1(6): 93-243.

Miguez, G.E., J.A. Funes Coronel y C.M. Gramajo Bühler

2009 Tecnología lítica en el piedemonte tucumano durante el Formativo. El caso de Horco Molle. La Zaranda de Ideas: Revista de Jóvenes Investigadores en Arqueología, 5: 133146.

Nelson, M.C.

1991 The Study of Technological Organization. Archaeological Method and Theory, 3: 57100. 
Palomo, A., R. Piqué, X. Terradas, O. López, I. Clemente y J.F. Gibaja

2013 Woodworking technology in the early neolithic site of La Draga (Banyoles, Spain). Regards croisés sur les outils liés au travail des végétaux, XXXIII Rencontres Internationales d'Archéologie et d'Histoire d'Antibes Sous (ed. por P. C. Anderson, C. Cheval y A. Durand), pp. 383-396. Éditions APDCA, Antibes.

Parry, W. J y R. Kelly

1987 Expedient core technology and sedentism. The Organization of Core Technology (ed. por J. Johnson y C. Morrow), pp. 285-304. Westview Press, Boulder y Londres. https://ci.nii.ac.jp/naid/10006683368/

Pérez, S.

2007 Aportes Metodológicos para el Análisis de Palas y/o Azadas Líticas. Mundo de Antes, 5: 73-89.

2010 Variabilidad en la producción de palas y/o azadas líticas de la Puna argentina. Estudios atacameños, 40: 5-22. https:// doi.org/10.4067/S0718-10432010000200002

2012 Tecnología lítica de la Puna meridional Argentina: Estrategias tecnológicas conservadas en la producción de artefactos agrícolas en contextos agropastoriles tempranos. Editorial Dunken, Buenos Aires.

Raffino, R.A.

2007 Poblaciones indígenas en Argentina: Urbanismo y proceso social precolombino. Emece Editores, Buenos Aires.

Salgán, L., \& Pompei, M.P.

2020 Las palas/azadas y/o cuñas líticas de cerro El Peceño (sur de Mendoza): una primera aproximación tecnológica. Revista del Museo de Antropología, 13(1): 223-228.

Seggiaro, R.E., P. Caffe, R.A. Becchio, C.I. Galli, M.J. Arnosio y G. Da Poian 2014 Evolución tectónica andina entre las sierras de Hualfín, Capillitas y extremo sur de Aconquija, Catamarca. Revista de La Asociación Geológica Argentina, 71(4): 500-512.

Sempé, M.C.

1999 La cultura Belén. Actas del XII Congreso Nacional de Arqueología Argentina, 2, pp. 250-258. Universidad Nacional de La Plata, La Plata.

2005 El Período Tardío en Azampay: El señorío Belén y su modelo geopolítico. Azampay: Presente y pasado de un pueblito catarmaqueño (ed. por M. C. Sempé, S. Salceda y M. M. Maffia), pp. 365-380. Ediciones Al Margen, La Plata.

Sonnenfeld, J.

1962 Interpreting the Function of Primitive Implements. American Antiquity, 28(1): 5665. https:// doi.org/10.2307/278078 
Sprovieri, M. y L. Baldini

2007 Aproximación a la producción lítica en sociedades tardías. El caso de Molinos I, valle Calchaquí Central (Salta). Intersecciones en antropología, 8: 135-147.

Valencia, M.C., N.I. Zagorodny, y S.M. Rivera

2009 Análisis de restos de madera del sitio Campo de Carrizal, Valle de Hualfín (Catamarca, Argentina). Darwiniana, 47(2): 260-266.

Xie, L.

2018 Scapulae for shovels: Does raw material choice reflect technological ease and low cost in production? Journal of Archaeological Science, 97: 77-89.

https://doi.org/10.1016/i.jas.2018.06.009

Yang, Y., W. Li, L. Yao, Z. Cheng, W. Luo, J. Zhang, L. Lin, H. Gan y L. Yan

2016 Plant food sources and stone tools' function at the site of Shunshanji based on starch grain analysis. Science China Earth Sciences, 59(8): 1574-1582.

https:// doi.org/10.1007/s11430-016-5321-9 